\title{
Operator identities in $q$-deformed Clifford analysis
}

\author{
K. Coulembier and F. Sommen
}

\begin{abstract}
In this paper, we define a $q$-deformation of the Dirac operator as a generalization of the one dimensional $q$-derivative. This is done in the abstract setting of radial algebra. This leads to a $q$-Dirac operator in Clifford analysis. The $q$-integration on $\mathbb{R}^{m}$, for which the $q$-Dirac operator satisfies Stokes' formula, is defined. The orthogonal $q$-CliffordHermite polynomials for this integration are briefly studied.
\end{abstract}

MSC 2000 : 30G35, 15A66,

Keywords : $q$-derivative, Dirac operator, radial algebra

\section{Introduction}

In [13] Jackson originally introduced the $q$-analogues of differentiation, integration and special functions in the context of $q$-hypergeometric series (also known as basic hypergeometric series). An overview of the theory of $q$-calculus can be found in $[9,10]$. In this paper we aim to define a $q$-deformation of the Dirac operator, which implies a $q$-deformation of partial derivatives in higher dimensions. Generalizations of the $q$-derivative to higher dimensions have also been developed in the theory of quantum spaces, see e.g. [2].

In [4] another approach to develop a $q$-Dirac operator was taken. In the present paper the behavior of the $q$-Dirac operator with respect to vectors plays a central role. It is therefore logical to define the $q$-Dirac operator in setting of radial algebra, see [15]. The $q$-Dirac operator on radial algebra is defined by a list of axioms based on $q$-calculus and Clifford analysis. It is then proven that this list of axioms uniquely defines a $q$-Dirac operator.

The $q$-Dirac operator on radial algebra leads to a $q$-Dirac operator in specific realizations of radial algebra, such as Clifford analysis or super Clifford analysis, see e.g. [6]. The expression for the $q$-Dirac operator in Clifford analysis shows that the deformation is in fact purely radial. An alternative theory of radial deformations of the Dirac operator is presented in [5]. For 
completeness we also derive the list of axioms that uniquely define the $q$ Dirac operator in Clifford analysis. Although the resulting $q$-Dirac operator differs from the one obtained in [4] the integration as defined in [4] also leads to Stokes' formula for the $q$-Dirac operator in the present paper.

Since the integration corresponds to the one in [4], the theory of orthogonal polynomials also coincides. Although the $q$-Dirac operator in the current paper is introduced more naturally, it seems that the theory of $q$-orthogonal polynomials in Clifford analysis is more elegantly described using the $q$-Dirac operator developed in [4].

This paper is organized as follows. First we recall the basic notions of $q$-calculus, Clifford analysis and radial algebra. The $q$-Dirac operator on radial algebra is defined by a list of axioms. Explicit calculations show that the axioms uniquely define the action of the $q$-Dirac operator on elements of the radial algebra of low degree. The applied techniques are then used to prove the unicity of the $q$-Dirac operator. Then it is proven that the list of axioms is not inconsistent and therefore uniquely defines a $q$-Dirac operator. This implies the definition of the $q$-Dirac operator in Clifford analysis. The action on terms of the Fischer decomposition is calculated and the unicity of the $q$-Dirac operator in Clifford analysis is proven. It turns out that the notion of $q$-monogenic polynomials and monogenic polynomials coincide, which implies that the deformation is purely radial. Then the $q$-integration on $\mathbb{R}^{m}$ is defined as $q$-deformed radial integration combined with undeformed spherical integration. It is proven that the $q$-Dirac operator satisfies Stokes' formula. Then it is argued that the $q$-Dirac operator as developed in [4] is better suited to study orthogonal polynomials. Finally in the conclusion the results are reviewed and a comparison is made between the $q$-Dirac operator in the current paper and the one in [4].

\section{Preliminaries}

First we give a short introduction to $q$-calculus, see e.g. $[9,10,13]$. We define for $u$ a number or operator, and the deformation parameter $q$,

$$
[u]_{q}=\frac{q^{u}-1}{q-1} .
$$

It's clear that $\lim _{q \rightarrow 1}[u]_{q}=u$. In this paper we always assume $0<q<1$.

The $q$-derivative of a function $f(t)$ is given by the expression

$$
D_{t}^{q}(f(t))=\frac{f(q t)-f(t)}{(q-1) t},
$$

which implies

$$
D_{t}^{q}\left(t^{k}\right)=\frac{q^{k}-1}{q-1} t^{k-1}=[k]_{q} t^{k-1}
$$

and

$$
D_{t}^{q} t=q t D_{t}^{q}+1 .
$$


The $q$-derivative satisfies the Leibniz rule

$$
D_{t}^{q}\left(f_{1}(t) f_{2}(t)\right)=D_{t}^{q}\left(f_{1}(t)\right) f_{2}(t)+f_{1}(q t) D_{t}^{q}\left(f_{2}(t)\right)
$$

The $q$-integration or Jackson integration on an interval $[0, a]$ with $a \in \mathbb{R}$ is given by

$$
\int_{0}^{a} f(t) d_{q} t=(1-q) a \sum_{k=0}^{\infty} f\left(a q^{k}\right) q^{k}
$$

The infinite $q$-integration is given by

$$
\int_{0}^{a \cdot \infty} f(t) d_{q} t=(1-q) a \sum_{k=-\infty}^{\infty} f\left(a q^{k}\right) q^{k}
$$

for $a \in \mathbb{R}$, see [11]. The infinite integration is therefore a function of $a$. However it is a $q$-constant, $D_{a}^{q} \int_{0}^{a \cdot \infty}=0$. More general intervals are defined by $\int_{a}^{b}=\int_{0}^{b}-\int_{0}^{a}$, and satisfy the important property

$$
\int_{a}^{b} D_{t}^{q} f(t) d_{q} t=f(b)-f(a) .
$$

There also exists an extensive theory of $q$-polynomials and -special functions, see e.g. $[9,12]$. For a general $\alpha>-1$ we define the $q$-Laguerre polynomials, see e.g. $[12,14]$ as

$$
\mathcal{L}_{t}^{\alpha}\left(u \mid q^{2}\right)=\sum_{i=0}^{t} q^{(t-i)(t-i+1)} \frac{(-u)^{i}}{[t-i]_{q^{2}} ![i]_{q^{2}} !} \frac{\Gamma_{q^{2}}(t+\alpha+1)}{\Gamma_{q^{2}}(i+\alpha+1)},
$$

with the $q$-factorial given by $[k]_{q} !=[k]_{q}[k-1]_{q} \cdots[1]_{q}$ and the $q$-Gamma function $\Gamma_{q}$ which satisfies $\Gamma_{q}(u+1)=u \Gamma_{q}(u)$. We also introduce a $q$-exponential by

$$
e_{q^{2}}(u)=\sum_{j=0}^{\infty} \frac{q^{j(j-1)} u^{j}}{[j]_{q^{2}} !} .
$$

Now we briefly recall the basic notions of Clifford analysis. For more details we refer the reader to $[1,8]$. The complex Clifford algebra $\mathbb{C}_{m}$ is generated by an orthonormal basis $\left(e_{1}, \cdots, e_{m}\right)$ for $\mathbb{R}^{m}$ with multiplication rules

$$
e_{i} e_{j}+e_{j} e_{i}=-2 \delta_{i j} \quad \text { for } \quad 1 \leq i, j \leq m .
$$

The algebra generated by these Clifford numbers and the $m$ variables $x_{j}$, which commute with the $e_{i}$, is the algebra of Clifford-valued polynomials $\mathcal{P}=\mathbb{R}\left[x_{1}, \cdots, x_{m}\right] \otimes \mathbb{C}_{m}$. The vector variable $\underline{x}$ on $\mathbb{R}^{m}$ can be identified with the first order Clifford polynomial of the form $\underline{x}=\sum_{j=1}^{m} e_{j} x_{j}$. The multiplication rules (7) imply that the square of this vector variable is scalar valued,

$$
\underline{x}^{2}=-\sum_{j=1}^{m} x_{j}^{2}=-r^{2}
$$


The corresponding Dirac operator or vector derivative in the vector variable $\underline{x}$ is the operator

$$
\partial_{\underline{x}}=-\sum_{j=1}^{m} e_{j} \partial_{x_{j}} .
$$

The square of the Dirac operator is again scalar and is the Laplace operator. Clifford analysis deals with the function theory of solutions of the equation $\partial_{\underline{x}} f(\underline{x})=0$, called monogenic functions. In particular we study monogenic polynomials of degree $k$. Denote by $\mathbb{E}=\sum_{j=1}^{m} x_{j} \partial_{x_{j}}$ the Euler operator.

Definition 1. An element $F \in \mathcal{P}$ is a spherical monogenic of degree $k$ if it satisfies $\partial_{\underline{x}} F=0$ and $\mathbb{E} F=k F$, i.e. $F \in \mathcal{P}_{k}$. Moreover the space of all spherical monogenics of degree $k$ is denoted by $\mathcal{M}_{k}$. follows.

The space of Clifford algebra-valued polynomials can be decomposed as

Lemma 1 (Fischer decomposition). The space $\mathcal{P}_{k}$ decomposes as

$$
\mathcal{P}_{k}=\bigoplus_{i=0}^{k} \underline{x}^{i} \mathcal{M}_{k-i}
$$

This decompositions is unique, so $\sum_{i} \underline{x}^{i} M_{k-i}=0$ (with $M_{k-i} \in \mathcal{M}_{k-i}$ ) implies $M_{k-i}=0$ for every $i$.

The role of the special orthogonal group $S O(m)$ in harmonic analysis is taken over by the spin group $\operatorname{Spin}(m)$ in Clifford analysis,

$$
\operatorname{Spin}(m)=\left\{s \in \mathbb{C}_{m} \mid \quad \exists k \in \mathbb{N}, s=\underline{\omega}_{1} \cdots \underline{\omega}_{2 k}, \quad \underline{\omega}_{i} \in \mathbb{S}^{m-1}, i=1, \cdots, 2 k\right\} .
$$

A real vector $\underline{\omega}$ in the Clifford algebra belongs to unit sphere $\mathbb{S}^{m-1}$ if $\underline{\omega}^{2}=-1$. The spin group is a double cover of the special orthogonal group. The $L$ representation of the spin group on Clifford algebra-valued functions is given by

$$
L(s)[f(\underline{x})]=s f\left(s^{-1} \underline{x} s\right) .
$$

The Dirac operator is is spin-invariant,

$$
\left[\partial_{\underline{x}}, L(s)\right]=0 .
$$

We will also need the main anti-involution ${ }^{-}$on the Clifford algebra $\mathbb{C}_{m}$, defined by

- - is equal to the complex conjugation on scalars,

- $\overline{e_{i}}=-e_{i}$,

- $\overline{a b}=\bar{b} \bar{a}$ for all $a, b \in \mathbb{C}_{m}$.

Using the Clifford algebra multiplication rules (7) yields

$$
\left\{\underline{x}, \partial_{\underline{x}}\right\}=\partial_{\underline{x}} \underline{x}+\underline{x} \partial_{\underline{x}}=2 \mathbb{E}+m .
$$

In particular the relations $\partial_{\underline{x}}(\underline{x})=m$ and

$$
\partial_{\underline{x}} \underline{x}^{2}=\underline{x}^{2} \partial_{\underline{x}}+2 \underline{x}
$$


hold.

The relation $\left\{\underline{x}, e_{j}\right\}=-2 x_{j}$ implies that $\{\underline{x}, \underline{z}\}$ is scalar valued for a general vector $\underline{z} \in \mathbb{R}^{m}$ identified with $\underline{z}=\sum_{j=1}^{m} e_{j} z_{j} \in \mathbb{C}_{m}$.

This leads us to radial algebra, see [15]. The starting object in the definition of radial algebra is a set $S$ of 'abstract vector variables'. In this paper we will always assume an infinite set $S$. The radial algebra $R(S)$ is the universal algabra generated by $S$ and subject to the constraints

$$
[\{x, y\}, z]=0 \quad \text { for any } \quad x, y, z \in S .
$$

The subset of $R(S)$ which commutes with all the elements of $R(S)$ is called the set of scalars, and denoted by $R_{0}(S)$. The formal inner product of two elements of the radial algebra is given by

$$
\langle u, v\rangle=\frac{1}{2}\{u, v\}
$$

and is an element of $R_{0}(S)$, by (11). In [15] it was proven that $R_{0}(S)$ is generated by the formal inner products. The space $R_{1}(S)$ is defined as the space of $R_{0}(S)$-linear combinations of elements of $S$. Clifford analysis is obtained again when we take the set $S=\left\{\underline{x}, e_{1}, \cdots, e_{m}\right\}$, therefore only viewing the 'prefered vector' $\underline{x}$ as a variable. Since this set $S$ is finite, not all results from radial algebra will be immediately applicable to Clifford analysis.

We can construct a vector derivative (Dirac operator) with respect to each element of $S$. Mostly we will choose $x$. This means that all the other operators, such as the Euler operator, are defined with respect to $x$. So we will always use the notation $\mathbb{E}$ in stead of $\mathbb{E}_{x}$. The subspace of the radial algebra $R(S)$ which is of degree $k$ with respect to $x$ is denoted by $[R(S)]_{k}$.

In order to define the vector derivative $\partial_{x} \in \operatorname{End}(R(S))$, there has to be a unique constant scalar $m$ for which

$$
\partial_{x}(x)=m, \quad \forall x \in S .
$$

On the level of radial algebra this $m$ is a parameter wich can take any value in $\mathbb{R}$. The Dirac operator is defined uniquely by this axiom and by

$$
\begin{aligned}
& \partial_{x}(f F)=\partial_{x}(f) F+f \partial_{x}(F), \quad f \in R_{0}(S), \quad F \in R(S) \\
& \partial_{x}(F G)=\partial_{x}(F) G, \quad F \in R(S), \quad G \in R(S \backslash\{x\}) \\
& \partial_{x}\left(x^{2}\right)=2 x \\
& \partial_{x}(\langle x, z\rangle)=z, \quad z \neq x .
\end{aligned}
$$

An important result of radial algebra is that it allows to develop a theory of super Clifford analysis, see e.g. [6]. Using $p$ commuting variables and $2 n$ anti-commuting variables we obtain a model for radial algebra with (super-)dimension $M=p-2 n$. So here the dimension of the radial algebra is an element of $\mathbb{Z}$. In [7] it was proven that there is a Fischer decomposition (lemma 1) in superspace if $M \notin-2 \mathbb{N}$. 


\section{The $q$-Dirac operator on radial algebra}

\subsection{Definition}

Our aim is to define a $q$-deformed vector derivative on the level of radial algebra. Property (2) implies that the following relation holds for the one dimensional $q$-derivative:

$$
D_{t}^{q} t^{2}=q^{2} t^{2} D_{t}^{q}+(q+1) t .
$$

Comparing this with property (10) we find that the $q$-Dirac operator should satisfy $\partial_{x}^{q} x^{2}=q^{2} x^{2} \partial_{x}^{q}+(q+1) x$. Formula (1) implies $D_{t}^{q}(t)=[1]_{q}$. Therefore we also impose $\partial_{x}^{q}(x)=[m]_{q}$ for some parameter $m \in \mathbb{R}$ and $\partial_{x}^{q}(\langle z, x\rangle)=\frac{[m]_{q}}{m} z$. We also assume $\partial_{x}^{q}$ to be vector valued, i.e. $\partial_{x}^{q}$ acting on $R_{0}(S)$ should be inside $R_{1}(S)$ and $\partial_{v}\left\langle v, \partial_{x}^{q}\right\rangle=\partial_{x}^{q}$ on $R(S \backslash\{v\})$ with $2\left\langle v, \partial_{x}^{q}\right\rangle=\left\{v, \partial_{x}^{q}\right\}$. In undeformed radial algebra the anticommutator of $x$ and $\partial_{x}$ commute with other vectors, see equation (9). It is logical to extend this property to the $q$-anticommutator of $x$ and $\partial_{x}^{q}$. Thus we are led to the following axioms for $\partial_{x}^{q} \in \operatorname{End}(R(S))$,

(B1) $\quad \partial_{x}^{q}(f) \in R_{1}(S), \quad f \in R_{0}(S)$ and $\partial_{v}\left\langle v, \partial_{x}^{q}\right\rangle=\partial_{x}^{q}$ on $R(S \backslash\{v\})$

(B2) $\quad \partial_{x}^{q}(F G)=\partial_{x}^{q}(F) G \quad F \in R(S), G \in R(S \backslash\{x\})$

(B3) $\quad \partial_{x}^{q} x^{2}=q^{2} x^{2} \partial_{x}^{q}+(q+1) x$

(B4) $\quad \partial_{x}^{q}(\langle x, z\rangle)=\frac{[m]_{q}}{m} z \quad \forall z \neq x$

(B5) $\quad\left[\left(\partial_{x}^{q} x+q x \partial_{x}^{q}\right), z\right]=0 \quad \forall z \neq x$

(B6) $\quad \lim _{z \rightarrow u} \partial_{x}^{q} F(z)=\partial_{x}^{q} F(u), \quad z \neq x \neq u$

$$
\mathbb{E} \partial_{x}^{q}=\partial_{x}^{q}(\mathbb{E}-1) \quad \text { and } \quad \mathbb{E}_{u} \partial_{x}^{q}=\partial_{x}^{q} \mathbb{E}_{u}, \quad u \neq x .
$$

Remark 1. It would seem logical to demand the relation $\partial_{x}^{q}\langle x, z\rangle=q\langle x, z\rangle \partial_{x}^{q}+$ $z$ as an axiom. However, this leads to a contradiction with axiom (B6) when we evaluate $\partial_{x}^{q}(\langle x, z\rangle\langle x, v\rangle)$.

\subsection{Unicity}

Now we prove some properties of an operator which satisfies axioms $(B 0)-$ (B7). This will lead to a proof of the unicity of the $q$-Dirac operator. The $q$-deformation of the Euler operator is given by

$$
E=[\mathbb{E}]_{q}=\frac{q^{\mathbb{E}}-1}{q-1}
$$

and satisfies

$$
E x-q x E=x \text { and } E z=z E .
$$

Lemma 2. An operator $\partial_{x}^{q} \in \operatorname{End}(R(S))$ satisfying axioms $(B 0),(B 3)$ and (B5) also satisfies the following q-deformed version of formula (9):

$$
\partial_{x}^{q} x+q x \partial_{x}^{q}=[m]_{q}+\left(q^{m}+q\right) E=[m+\mathbb{E}]_{q}+q[\mathbb{E}]_{q} .
$$


Proof. The combination of axioms $(B 2)$ and $(B 7)$ imply $\partial_{x}^{q}(1)=0$. We define the operator $A$ on $R(S)$ as

$$
A=\partial_{x}^{q} x+q x \partial_{x}^{q}-[m]_{q}-\left(q^{m}+q\right) E .
$$

Axiom $(B 0)$ and the relation $\partial_{x}^{q}(1)=0$ imply $A(1)=0$. Using the definition of $A$ and equation (12) we calculate

$$
\begin{aligned}
\partial_{x}^{q} x^{2} & =\left(A-q x \partial_{x}^{q}+[m]_{q}+\left(q^{m}+q\right) E\right) x \\
& =\left(A+[m]_{q}+\left(q^{m}+q\right) E\right) x-q x\left(A-q x \partial_{x}^{q}+[m]_{q}+\left(q^{m}+q\right) E\right) \\
& =A x-q x A+q^{2} x^{2} \partial_{x}^{q}+[m]_{q}(1-q) x+\left(q^{m}+q\right)(E x-q x E) \\
& =A x-q x A+q^{2} x^{2} \partial_{x}^{q}+(q+1) x .
\end{aligned}
$$

Comparing this with axiom (B3) yields $A x=q x A$. Using axiom (B5) and equation (12) we also find $A z=z A \forall z \neq x$, so $A=0$ as an operator on $R(S)$.

We will also need the following calculation.

Lemma 3. An operator $\partial_{x}^{q} \in \operatorname{End}(R(S))$ satisfying axiom (B5) also satisfies

$$
\partial_{x}^{q}\langle x, z\rangle-q\langle x, z\rangle \partial_{x}^{q}=\left\langle z, \partial_{x}^{q}\right\rangle x-q x\left\langle z, \partial_{x}^{q}\right\rangle .
$$

Proof. We calculate, using (B5)

$$
\begin{aligned}
2 \partial_{x}^{q}\langle x, z\rangle+2 q x\left\langle z, \partial_{x}^{q}\right\rangle & =\partial_{x}^{q} x z+q x \partial_{x}^{q} z+\partial_{x}^{q} z x+q x z \partial_{x}^{q} \\
& =z \partial_{x}^{q} x+z q x \partial_{x}^{q}+\partial_{x}^{q} z x+q x z \partial_{x}^{q} \\
& =2\left\langle z, \partial_{x}^{q}\right\rangle x+2 q\langle x, z\rangle \partial_{x}^{q}
\end{aligned}
$$

which gives the desired result.

Now we calculate some explicit evaluations of the Dirac operator. Because of lemma 3 and axiom (B4) we find

$$
\left\langle z, \partial_{x}^{q}\right\rangle(x)=\frac{[m]_{q}}{m} z \quad \text { and } \quad\left\langle z, \partial_{x}^{q}\right\rangle(\langle x, u\rangle)=\frac{[m]_{q}}{m}\langle z, u\rangle .
$$

Therefore all the first order evaluations are completely determined from axioms $(B 0)-(B 7)$. Now we consider second order evaluations. Axiom (B3) implies

$$
\partial_{x}^{q}\left(x^{2}\right)=(q+1) x
$$

and $\left\langle z, \partial_{x}^{q}\right\rangle\left(x^{2}\right)=(q+1)\langle x, z\rangle$. Equation (13) combined with axiom $(B 4)$ yields

$$
\partial_{x}^{q}(x\langle x, z\rangle)=-q x \frac{[m]_{q}}{m} z+\left([m+1]_{q}+q\right)\langle x, z\rangle .
$$

In order to find the other second order evaluations, we use a technique that will be generalized in the proof of theorem 1 . 
Lemma 4. Consider $z, u \in S$, different from $x$. For an operator $\partial_{x}^{q} \in \operatorname{End}(R(S))$ satisfying axioms $(B 0)-(B 7)$, the following relations hold:

$\partial_{x}^{q}(\langle x, z\rangle\langle x, u\rangle)=\frac{[m+1]_{q}+q}{m+2}(u\langle x, z\rangle+z\langle x, u\rangle)+\frac{1+q-2 q[m]_{q} / m}{m+2} x\langle u, z\rangle$

and

$\left\langle z, \partial_{x}^{q}\right\rangle(x\langle x, u\rangle)=\frac{[m+1]_{q}+q}{m+2}(x\langle u, z\rangle+z\langle x, u\rangle)+\frac{1+q-2 q[m]_{q} / m}{m+2} u\langle x, z\rangle$.

Proof. We start by using lemma 3 on $\partial_{x}^{q}(\langle x, u\rangle\langle x, z\rangle)$,

$$
\partial_{x}^{q}(\langle x, z\rangle\langle x, u\rangle)=q \frac{[m]_{q}}{m}\langle x, z\rangle u+\left\langle z, \partial_{x}^{q}\right\rangle(x\langle x, u\rangle)-q \frac{[m]_{q}}{m} x\langle z, u\rangle .
$$

So it suffices to calculate $\partial_{x}^{q}(\langle x, z\rangle\langle x, u\rangle)$. Axioms $(B 7)$ and $(B 1)$ imply that there must be coefficients $\alpha, \gamma$ and $\beta$ such that

$$
\partial_{x}^{q}(\langle x, z\rangle\langle x, u\rangle)=\alpha z\langle x, u\rangle+\gamma u\langle x, z\rangle+\beta x\langle z, u\rangle
$$

holds. The symmetry between $z$ and $u$ (axiom (B6)) implies $\alpha=\gamma$. Consider $v \in S$ and different from $x, u, z$, the calculation above implies

$$
\begin{aligned}
\left\langle v, \partial_{x}^{q}\right\rangle(\langle x, z\rangle\langle x, u\rangle) & =\alpha(\langle v, z\rangle\langle x, u\rangle+\langle v, u\rangle\langle x, z\rangle)+\beta\langle v, x\rangle\langle z, u\rangle \text { and } \\
\left\langle v, \partial_{x}^{q}\right\rangle(x\langle x, u\rangle) & =\alpha(v\langle x, u\rangle+\langle v, u\rangle x)+\beta\langle v, x\rangle u .
\end{aligned}
$$

Axiom (B1) then implies

$$
\begin{aligned}
\partial_{x}^{q} x\langle x, u\rangle & =\partial_{v}\left\langle v, \partial_{x}^{q}\right\rangle x\langle x, u\rangle \\
& =\alpha(m\langle x, u\rangle+u x)+\beta x u .
\end{aligned}
$$

Comparing this with formula (15), yields $\alpha=\frac{[m+1]_{q}+q}{m+2}$ and $\beta=\frac{1+q-2 q[m]_{q} / m}{m+2}$.

This lemma and the calculation before imply that the operator $\partial_{x}^{q}$ is uniquely determined by axioms $(B 0)-(B 7)$ on the space $[R(S)]_{0} \oplus[R(S)]_{1} \oplus$ $[R(S)]_{2}$. By generalizing the used techniques we can prove the unicity on $R(S)$. First the symmetry is used in the following lemma to limit the possible expressions for $\partial_{x}^{q}$ acting on elements of $R(S)$. We use the convention that the notation $\left\langle x, u_{i}\right\rangle\left\langle x, u_{i+1}\right\rangle \cdots\left\langle x, u_{k}\right\rangle$ is considered to be 1 if $k<i$ and introduce some notations. For $u_{i}$ elements of $S$ and $S_{j-1}$ the permutation group on $\{1,2, \cdots, j-1\}$, define the element of $R_{0}(S)$ given by

$$
=\sum_{\sigma \in S_{j-1}}\left\langle u_{\sigma(1)}^{j}, u_{\sigma(2)}\right\rangle \cdots\left\langle u_{\sigma(2 i-1)}, u_{\sigma(2 i)}\right\rangle\left\langle x, u_{\sigma(2 i+1)}\right\rangle \cdots\left\langle x, u_{\sigma(j-1)}\right\rangle
$$

and the element of $R_{1}(S)$ given by

$$
=\sum_{\sigma \in S_{j-1}}^{\Pi_{i}^{j}\left(u_{1}, \cdots, u_{j-1}\right)} u_{\sigma(1)}\left\langle u_{\sigma(2)}, u_{\sigma(3)}\right\rangle \cdots\left\langle u_{\sigma(2 i)}, u_{\sigma(2 i+1)}\right\rangle\left\langle x, u_{\sigma(2 i+2)}\right\rangle \cdots\left\langle x, u_{\sigma(j-1)}\right\rangle .
$$


The following properties of $\Lambda$ and $\Pi$ are straightforward to obtain

$$
\Pi_{i}^{j} x=2 \Lambda_{i}^{j}-x \Pi_{i}^{j}
$$

and

$$
\begin{aligned}
& \Lambda_{i}^{j}\left(u_{1}, \cdots, u_{j-1}\right)=\sum_{\sigma \in S_{j-1}} u_{\sigma(1)} u_{\sigma(2)}\left\langle u_{\sigma(3)}, u_{\sigma(4)}\right\rangle \cdots \\
& \cdots\left\langle u_{\sigma(2 i-1)}, u_{\sigma(2 i)}\right\rangle\left\langle x, u_{\sigma(2 i+1)}\right\rangle \cdots\left\langle x, u_{\sigma(j-1)}\right\rangle .
\end{aligned}
$$

Lemma 5. With $x, v, u_{1}, u_{2}, \cdots, u_{j}$ all different from each other and $\partial_{x}^{q}$ an operator satisfying axioms $(B 0)-(B 7)$, the expression

$$
\left\langle v, \partial_{x}^{q}\right\rangle\left(\left\langle x, u_{1}\right\rangle\left\langle x, u_{2}\right\rangle \cdots\left\langle x, u_{j}\right\rangle\right)
$$

has to be of the following form

$$
\sum_{i=0}^{\left\lfloor\frac{j-1}{2}\right\rfloor} \alpha_{i} x^{2 i}\left\langle v, \Pi_{i}^{j+1}\left(u_{1}, \cdots, u_{j}\right)\right\rangle+\sum_{i=0}^{\left\lfloor\frac{j-2}{2}\right\rfloor} \beta_{i} x^{2 i}\langle v, x\rangle \Lambda_{i+1}^{j+1}\left(u_{1}, \cdots, u_{j}\right)
$$

for some constants $\left\{\alpha_{i}\right\}$ and $\left\{\beta_{i}\right\}$.

Proof. The fact that every term is accompanied by permutations of the $u_{l}$ is immediately clear from the fact that $u_{1}, \cdots u_{j}$ all have the same indistinguishable role, as implied by axiom (B6). Axioms (B1) and (B7) imply that

$$
\left\langle v, \partial_{x}^{q}\right\rangle\left(\left\langle x, u_{1}\right\rangle\left\langle x, u_{2}\right\rangle \cdots\left\langle x, u_{j}\right\rangle\right)
$$

is an element of $R_{0}(S)$ of degree $j-1$ in $x$ and of degree 1 in $v$ and $u_{l}$ for $1 \leq l \leq j$.

First we consider all the possible terms without an $x^{2}$, these correspond to $i=0$ in the summation above. So we need to make inner products of $x$ with $j-1$ of the $v, u_{1}, \cdots, u_{j}$. Besides these inner products with $x$, there either has to be an inner product $\left\langle v, u_{\lambda}\right\rangle$ or an inner product of the form $\left\langle u_{\lambda}, u_{\rho}\right\rangle$. For the first option to be possible, $j \geq 1$ has to hold. For the second option to be possible, $j \geq 2$ has to hold. These are the two possibilities that correspond to $\alpha_{0}$ and $\beta_{0}$. A completely similar reasoning leads to the terms with $i \geq 1$.

Now lemma 4 can be generalized to arbitrary degrees.

Theorem 1. For an operator $\partial_{x}^{q} \in \operatorname{End}(R(S))$ satisfying axioms $(B 0)-(B 7)$, the expression

$$
\partial_{x}^{q}\left\langle x, u_{1}\right\rangle\left\langle x, u_{2}\right\rangle \cdots\left\langle x, u_{j}\right\rangle
$$

with all the $u_{i}$ different from each other and from $x$, is completely determined for $j \in \mathbb{N}$.

Proof. This expression is determined for $j=1$, by axiom $(B 4)$. Now, define

$$
F_{j}\left(u_{1}, u_{2}, \cdots, u_{j}\right)=\partial_{x}^{q}\left\langle x, u_{1}\right\rangle\left\langle x, u_{2}\right\rangle \cdots\left\langle x, u_{j}\right\rangle .
$$


Lemma 5 shows which form $\left\langle v, F_{j}\right\rangle$ takes. This implies that

$$
\begin{aligned}
& F_{j}\left(u_{1}, u_{2}, \cdots, u_{j}\right) \\
= & \sum_{i=0}^{\left\lfloor\frac{j-1}{2}\right\rfloor} \alpha_{i}^{j} x^{2 i} \Pi_{i}^{j+1}\left(u_{1}, \cdots, u_{j}\right)+\sum_{i=0}^{\left\lfloor\frac{j-2}{2}\right\rfloor} \beta_{i}^{j} x^{2 i+1} \Lambda_{i+1}^{j+1}\left(u_{1}, \cdots, u_{j}\right)
\end{aligned}
$$

for some coefficients $\alpha$ and $\beta$. Now we will prove that the coefficients $\left\{\alpha_{i}^{j}, i \leq\right.$ $\left.\left\lfloor\frac{j-1}{2}\right\rfloor\right\}$ and $\left\{\beta_{i}^{j}, i \leq\left\lfloor\frac{j-2}{2}\right\rfloor\right\}$ are determined in terms of the coefficients $\left\{\alpha_{i}^{j-1}, i \leq\left\lfloor\frac{j-2}{2}\right\rfloor\right\}$ and $\left\{\beta_{i}^{j-1}, i \leq\left\lfloor\frac{j-3}{2}\right\rfloor\right\}$. This proves by induction that the coefficients are determined, since they are known for $j=1$. with

Define $G_{j}\left(u_{1}, u_{2}, \cdots, u_{j-1}\right)$ by $\left\langle G_{j}, u_{j}\right\rangle=\left\langle v, F_{j}\right\rangle$, which is equivalent

$$
G_{j}=\left\langle v, \partial_{x}^{q}\right\rangle\left\langle x, u_{1}\right\rangle \cdots\left\langle x, u_{j-1}\right\rangle x
$$

From the expression of $\left\langle v, F_{j}\right\rangle$ we can calculate $G_{j}$,

$$
\begin{aligned}
& G_{j}\left(u_{1}, u_{2}, \cdots, u_{j-1}\right)=\sum_{i=0}^{\left\lfloor\frac{j-1}{2}\right\rfloor} \alpha_{i}^{j} x^{2 i} v \Lambda_{i}^{j}\left(u_{1}, \cdots, u_{j-1}\right) \\
& +\sum_{i=0}^{\left\lfloor\frac{j-1}{2}\right\rfloor} \alpha_{i}^{j} x^{2 i} 2 i\left[\sum_{\sigma \in S_{j-1}}\left\langle v, u_{\sigma(1)}\right\rangle u_{\sigma(2)}\left\langle u_{\sigma(3)}, u_{\sigma(4)}\right\rangle \cdots\right. \\
& \left.\cdots\left\langle u_{\sigma(2 i-1)}, u_{\sigma(2 i)}\right\rangle\left\langle x, u_{\sigma(2 i+1)}\right\rangle \cdots\left\langle x, u_{\sigma(j-1)}\right\rangle\right] \\
& +\sum_{i=0}^{\left\lfloor\frac{j-1}{2}\right\rfloor} \alpha_{i}^{j} x^{2 i+1}(j-2 i-1)\left\langle v, \Pi_{i}^{j}\left(u_{1}, \cdots, u_{j-1}\right)\right\rangle \\
& +\sum_{i=0}^{\left\lfloor\frac{j-2}{2}\right\rfloor} \beta_{i}^{j} x^{2 i}(2 i+2)\langle v, x\rangle \Pi_{i}^{j}\left(u_{1}, \cdots, u_{j-1}\right) \\
& +\sum_{i=0}^{\left\lfloor\frac{j-2}{2}\right\rfloor} \beta_{i}^{j} x^{2 i+1}(j-2 i-2)\langle v, x\rangle \Lambda_{i+1}^{j}\left(u_{1}, \cdots, u_{j-1}\right) .
\end{aligned}
$$

This allows to calculate $\partial_{v} G_{j}$ using equations (17) and (18),

$$
\begin{gathered}
\partial_{v} G_{j}\left(u_{1}, u_{2}, \cdots, u_{j-1}\right)=\sum_{i=0}^{\left\lfloor\frac{j-1}{2}\right\rfloor} \alpha_{i}^{j} x^{2 i}(m+2 i) \Lambda_{i}^{j}\left(u_{1}, \cdots, u_{j-1}\right) \\
+\sum_{i=0}^{\left\lfloor\frac{j-1}{2}\right\rfloor} \alpha_{i}^{j} x^{2 i}(j-2 i-1)\left[2 \Lambda_{i}^{j}\left(u_{1}, \cdots, u_{j-1}\right)-x \Pi_{i}^{j}\left(u_{1}, \cdots, u_{j-1}\right)\right]
\end{gathered}
$$




$$
\begin{aligned}
& +\sum_{i=0}^{\left\lfloor\frac{j-2}{2}\right\rfloor} \beta_{i}^{j} x^{2 i+1}(2 i+2) \Pi_{i}^{j}\left(u_{1}, \cdots, u_{j-1}\right) \\
& +\sum_{i=0}^{\left\lfloor\frac{j-2}{2}\right\rfloor} \beta_{i}^{j} x^{2 i+2}(j-2 i-2) \Lambda_{i+1}^{j}\left(u_{1}, \cdots, u_{j-1}\right) .
\end{aligned}
$$

By combining terms and taking into account zero terms we obtain

$$
\begin{aligned}
& \partial_{v} G_{j}\left(u_{1}, u_{2}, \cdots, u_{j-1}\right) \\
= & (m+2 j-2) \alpha_{0}^{j}(j-1) !\left\langle x, u_{1}\right\rangle \cdots\left\langle x, u_{j-1}\right\rangle \\
+ & \sum_{i=1}^{\left\lfloor\frac{j-1}{2}\right\rfloor}\left[(m+2 j-2 i-2) \alpha_{i}^{j}+(j-2 i) \beta_{i-1}^{j}\right] x^{2 i} \Lambda_{i}^{j}\left(u_{1}, \cdots, u_{j-1}\right) \\
+ & \sum_{i=0}^{\left\lfloor\frac{j-2}{2}\right\rfloor}\left[(2 i+2) \beta_{i}^{j}-(j-2 i-1) \alpha_{i}^{j}\right] x^{2 i+1} \Pi_{i}^{j}\left(u_{1}, \cdots, u_{j-1}\right) .
\end{aligned}
$$

The expression $\partial_{v} G_{j}$ can also be calculated starting from equation (19), using axiom $(B 1)$, the expression of $F_{j-1}$ in terms of $\Lambda$ and $\Pi$ and lemma 2 ,

$$
\begin{aligned}
& \partial_{v} G_{j} \\
= & \partial_{x}^{q} x\left\langle x, u_{1}\right\rangle \cdots\left\langle x, u_{j-1}\right\rangle \\
= & \left([m+j-1]_{q}+q[j-1]_{q}\right)\left\langle x, u_{1}\right\rangle \cdots\left\langle x, u_{j-1}\right\rangle \\
- & \sum_{i=0}^{\left\lfloor\frac{j-2}{2}\right\rfloor} q \alpha_{i}^{j-1} x^{2 i+1} \Pi_{i}^{j}\left(u_{1}, \cdots, u_{j-1}\right)-\sum_{i=0}^{\left\lfloor\frac{j-3}{2}\right\rfloor} q \beta_{i}^{j-1} x^{2 i+2} \Lambda_{i+1}^{j}\left(u_{1}, \cdots, u_{j-1}\right)
\end{aligned}
$$

Comparing coefficients yields

$$
\begin{aligned}
\alpha_{0}^{j} & =\frac{[m+j-1]_{q}+q[j-1]_{q}}{(m+2 j-2)(j-1) !}, \\
{\left[(m+2 j-2 i-2) \alpha_{i}^{j}+(j-2 i) \beta_{i-1}^{j}\right] } & =-q \beta_{i-1}^{j-1} \text { and } \\
{\left[(2 i+2) \beta_{i}^{j}-(j-2 i-1) \alpha_{i}^{j}\right] } & =-q \alpha_{i}^{j-1} .
\end{aligned}
$$

Assuming that the coefficients for $j-1$ are known, this can clearly be solved by using the third equation to calculate $\beta_{0}^{j}$, then the second to calculate $\alpha_{1}^{j}$ and so forth. The solution will always be unique. However it will only exist if $m \notin-2 \mathbb{N}$.

Now we can prove the unicity of the $q$-Dirac operator if the parameter $m$ is not even and negative. If $m \in-2 \mathbb{N}$ the axioms are not consistent as can be seen from the proof of theorem 1 .

Theorem 2. There can only be one $q$-Dirac operator $\partial_{x}^{q} \in \operatorname{End}(R(S))$ satisfying axioms $(B 0)-(B 7)$. 
Proof. We need to prove that $\partial_{x}^{q}$ acting on all elements of $R(S)$ is determined from axioms $(B 0)-(B 7)$. The radial algebra $R(S)$ is additively generated by elements of the form

$$
x^{k}\left\langle x, u_{1}\right\rangle\left\langle x, u_{2}\right\rangle \cdots\left\langle x, u_{j}\right\rangle y_{1} \cdots y_{l},
$$

with $k \in \mathbb{N}, u_{i}, y_{i} \in S$ and different from $x$. Because of axiom (B6) we can consider all the $u_{i}$ to be different from each other. Lemma 2 and axiom (B2) imply that the $q$-Dirac operator acting on such elements of $R(S)$ is determined if the $q$-Dirac operator on $\left\langle x, u_{1}\right\rangle\left\langle x, u_{2}\right\rangle \cdots\left\langle x, u_{j}\right\rangle$ is determined. Theorem 1 therefore proves this theorem.

It should still be proven that the axioms $(B 0)-(B 7)$ are in general not inconsistent, this will be done in section 3.3 by explicit construcion of $\partial_{x}^{q}$ if $m \notin-2 \mathbb{N}$.

Remark 2. The calculations in theorem 1 imply that there cannot be a qdeformed Dirac operator in models of radial algebra for super Clifford analysis if the superdimension $M=p-2 n$ (with $p$ bosonic and $n$ fermionic variables) is even and negative. This corresponds to the case without Fischer decomposition, see [7].

We call an element $P$ of the radial algebra $q$-monogenic if it satisfies $\partial_{x}^{q} P=0$. Here we will find that a certain type of second degree polynomial is $q$-monogenic if it is monogenic. In section 3.3 we will find that every monogenic polynomial is $q$-monogenic.

Lemma 6. If the element of the radial algebra,

$$
\langle x, z\rangle^{2}+a\langle x, z\rangle x z+b x^{2} z^{2}
$$

with $a, b \in \mathbb{R}$ is monogenic, it is also $q$-monogenic if $m \notin-2 \mathbb{N}$.

Proof. Using the calculations in equations (14) and (15) and in lemma 4 we find

$$
\begin{aligned}
& \partial_{x}^{q}\left(\langle x, z\rangle^{2}+a\langle x, z\rangle x z+b x^{2} z^{2}\right) \\
= & \left(\frac{[m+1]_{q}+q}{m+2}\right) 2 z\langle x, z\rangle+\frac{1+q-2 q[m]_{q} / m}{m+2} x z^{2} \\
+ & a\left([m+1]_{q}+q\right)\langle x, z\rangle z-a q \frac{[m]_{q}}{m} x z^{2}+b(q+1) x z^{2} .
\end{aligned}
$$

Therefore it is $q$-monogenic if $a=\frac{-2}{m+2}$ and $b=\frac{-1}{m+2}$. This is independent from $q$ and therefore the polynomial is $q$-monogenic if it is monogenic.

In case the parameter $m$ satisfies $[m+1]_{q}+q=0$ (which requires $m$ to be negative) the element of $R(S)$ above is $q$-monogenic for all $(a, b)$ such that $1+a+m b=0$ holds. So for specific values of $q$ and $m$ the space of $q$-monogenic elements of $R(S)$ can include elements which are not monogenic. 


\subsection{Existence}

We give an explicit expression for $\partial_{x}^{q}$ in case $m \notin-2 \mathbb{N}$ in terms of the classical vector derivative $\partial_{x}$ defined by axioms $(A 0)-(A 4)$. In this way we prove that the set of axioms $(B 0)-(B 7)$ is consistent, and complete the unicity and existence of an operator defined by $(B 0)-(B 7)$. This explicit expression for $\partial_{x}^{q}$ in terms of $\partial_{x}$ also leads to a definition for the $q$-deformed Dirac operator in (super) Clifford analysis.

Theorem 3. If $m \notin-2 \mathbb{N}$ there is exactly one operator $\partial_{x}^{q}$ in $\operatorname{End}(R(S))$ which satisfies axioms $(B 0)-(B 7)$. The operator is given by

$$
\partial_{x}^{q}=\sum_{j=0}^{\infty} x^{j} f_{j}(\mathbb{E}) \partial_{x}^{j+1},
$$

with, for $j \in \mathbb{N}$, the functions $f_{j}: \mathbb{N} \rightarrow \mathbb{R}$ defined by $f_{0}(u)=\frac{[m+u]_{q}+q[u]_{q}}{m+2 u}$ and

$$
\begin{aligned}
f_{2 j+1}(u) & =\frac{1}{2 j+2}\left[f_{2 j}(u+1)-q f_{2 j}(u)\right] \text { and } \\
f_{2 j+2}(u) & =-\frac{1}{2 u+2 j+m+2}\left[f_{2 j+1}(u+1)+q f_{2 j+1}(u)\right] .
\end{aligned}
$$

Proof. The operator $f_{j}(\mathbb{E})$ is defined as the diagonal operator on $R(S)$ which has the same eigenvectors as $\mathbb{E}$ but with eigenvalues $k \in \mathbb{N}$ replaced by $f_{j}(k)$. Since each element of $R(S)$ is of finite maximal degree in $x$, the expression above for $\partial_{x}^{q}$ is an element of $\operatorname{End}(R(S))$.

Because of the unicity of an operator which satisfies axioms $(B 0)-(B 7)$, we only have to check that the proposed form of $\partial_{x}^{q}$ satisfies the axioms. Axioms $(B 0)$ and $(B 4)$ hold since $f_{0}(0)=[m]_{q} / m$. Because $x$ and $\partial_{x}$ are vector valued operators axiom $(B 1)$ holds. Axioms $(B 2),(B 6)$ and $(B 7)$ trivially hold. Now we will prove that equation (13) holds, from which (B3) and (B5) follow. Substituting the proposed expression for $\partial_{x}^{q}$ and using the formulas $\left\{\partial_{x}^{2 j+1}, x\right\}=\partial_{x}^{2 j}(2 \mathbb{E}+m)$ and $\left[\partial_{x}^{2 j}, x\right]=2 j \partial_{x}^{2 j-1}$ yields

$$
\begin{aligned}
\partial_{x}^{q} x+q x \partial_{x}^{q} & =-\sum_{j=0}^{\infty} x^{2 j+1} f_{2 j}(\mathbb{E}+1) \partial_{x}^{2 j+1}+\sum_{j=0}^{\infty} x^{2 j} f_{2 j}(\mathbb{E})(2 \mathbb{E}+m+2 j) \partial_{x}^{2 j} \\
& +\sum_{j=0}^{\infty} x^{2 j+2} f_{2 j+1}(\mathbb{E}+1) \partial_{x}^{2 j+2}+\sum_{j=0}^{\infty} x^{2 j+1}(2 j+2) f_{2 j+1}(\mathbb{E}) \partial_{x}^{2 j+1} \\
& +\sum_{j=0}^{\infty} x^{2 j+1} q f_{2 j}(\mathbb{E}) \partial_{x}^{2 j+1}+\sum_{j=0}^{\infty} x^{2 j+2} q f_{2 j+1}(\mathbb{E}) \partial_{x}^{2 j+2} \\
& =f_{0}(\mathbb{E})(2 \mathbb{E}+m)=[m+\mathbb{E}]_{q}+q[\mathbb{E}]_{q},
\end{aligned}
$$

which proves the theorem.

Remark 3. The explicit expression in theorem 3 implies that lemma 6 can be generalized immediately. Every monogenic polynomial is q-monogenic. The other direction is not true, a polynomial can be q-monogenic for specific values of $m$ and $q$ without being monogenic. 
Example 1. As an easy example we can calculate the expression for $\partial_{x}^{q}$ acting on elements of $R(S)$ of degree 2 in $x$,

$$
\left.\partial_{x}^{q}\right|_{[R(S)]_{2}}=\frac{[m+1]_{q}+q}{m+2} \partial_{x}+\frac{1}{2} \frac{1+q-2 q[m]_{q} / m}{m+2} x \partial_{x}^{2} .
$$

From this we immediately re-obtain the expressions in lemma 4.

\section{The $q$-Dirac operator in Clifford analysis}

The expression for the $q$-Dirac operator in terms of $\partial_{x}$ in theorem 3 allows to construct it for specific models of radial algebra. For Clifford analysis on $\mathbb{R}^{m}$, the $q$-Dirac operator on $\mathcal{P}=\mathbb{R}\left[x_{1}, \cdots, x_{m}\right] \otimes \mathbb{C}_{m}$ is therefore defined as

$$
\partial_{\underline{x}}^{q}=\sum_{j=0}^{\infty} \underline{x}^{j} f_{j}(\mathbb{E}) \partial_{\underline{x}}^{j+1},
$$

with $f_{j}$ the functions in theorem 3 . Although this expression is not very transparent, the action on terms of the Fischer decomposition can be calculated easily. This operator can also be determined uniquely by a list of axioms, closely related to the $B$-axioms. The only essential change is axiom $(B 6)$, which does not have the same implications in Clifford analysis, since the set $S$ is finite for Clifford analysis. Therefore it is replaced by the condition of $\operatorname{Spin}(m)$-invariance.

\subsection{The $q$-Dirac operator and the Fischer decompostion}

It is straightforward to check that the Dirac operator in Clifford analysis (20) still satisfies the relation

$$
\partial_{\underline{x}}^{q} \underline{x}+q \underline{x} \partial_{\underline{x}}^{q}=[m+\mathbb{E}]_{q}+q[\mathbb{E}]_{q}
$$

which also implies $\partial_{\underline{x}}^{q} \underline{x}^{2}=q^{2} \underline{x}^{2} \partial_{\underline{x}}^{q}+(q+1) \underline{x}$. The $q$-deformed Euler operator also satisfies $[\mathbb{E}]_{q}=\bar{r} D_{r}^{q}$. In Clifford analysis (with dimension $m>0$ ) we can prove that a polynomial is monogenic if and only if it is $q$-monogenic (with $q$ an arbitrary fixed constant satisfying $0<q<1$ ), see the discussion in remark 3 .

Theorem 4. Given $M_{k} \in \mathcal{M}_{k}$ a spherical monogenic of degree $k$, then the following relations holds:

$$
\begin{aligned}
\partial_{\underline{x}}^{q} \underline{x}^{2 l} M_{k} & =[2 l]_{q} \underline{x}^{2 l-1} M_{k} \quad l \in \mathbb{N}, \\
\partial_{\underline{x}}^{q} \underline{x}^{2 l+1} M_{k} & =\left([2 l+k+m]_{q}+q^{2 l+1}[k]_{q}\right) \underline{x}^{2 l} M_{k} \quad l \in \mathbb{N} .
\end{aligned}
$$

Given $P_{k} \in \mathcal{P}_{k}$ a polynomial of degree $k, \partial_{x}^{q} P_{k}=0$ holds if and only if $P_{k} \in \mathcal{M}_{k}$. 
Proof. The expression (20) immediately implies that $\partial_{\underline{x}}^{q} M_{k}=0$ if $M_{k} \in \mathcal{M}_{k}$. Applying the commutation relation (21) yields

$$
\begin{aligned}
\partial_{\underline{x}}^{q} \underline{x}^{2 l} M_{k} & =\left(q^{2 l} x^{2 l} \partial_{\underline{x}}^{q}+\frac{q^{2 l}-1}{q-1} \underline{x}^{2 l-1}\right) M_{k} \\
& =[2 l]_{q} \underline{x}^{2 l-1} M_{k}
\end{aligned}
$$

and

$$
\begin{aligned}
\partial_{\underline{x}}^{q} \underline{x}^{2 l+1} M_{k} & =\left[q^{2 l} \underline{x}^{2 l} \partial_{\underline{x}}^{q} \underline{x}+\frac{q^{2 l}-1}{q-1} \underline{x}^{2 l}\right] M_{k} \\
& =\left[q^{2 l} \underline{x}^{2 l}\left([m+k]_{q}+q[k]_{q}\right)+[2 l]_{q}\right] \underline{x}^{2 l} M_{k} \\
& =\left([2 l+m+k]_{q}+q^{2 l+1}[k]_{q}\right) \underline{x}^{2 l} M_{k},
\end{aligned}
$$

which completes the proof of the first part of the theorem.

The obtained relations can be summarized as $\partial_{x}^{q} \underline{x}^{l} M_{k}=c_{l, k} \underline{x}^{l-1} M_{k}$ for $l>0$. The coefficients $c_{k, l}$ are strictly positive since $m>0, l>0$ and $k \geq 0$. Consider a fixed polynomial $P_{k} \in \mathcal{P}_{k}$ with Fischer decomposition (lemma 1)

$$
P_{k}=\sum_{j=0}^{k} \underline{x}^{j} M_{k-j} \Rightarrow \partial_{\underline{x}}^{q} P_{k}=\sum_{j=1}^{k} c_{j, k-j} \underline{x}^{j-1} M_{k-j} .
$$

The unicity of the Fischer decomposition then implies that if $P_{k}$ satisfies $\partial_{x}^{q} P_{k}=0$, then $M_{j}=0$ must hold for $j<k$ and therefore $P_{k}=M_{k}$ holds. This completes the proof.

The fact that the space of spherical $q$-monogenics is independent of $q$, shows that the only real $q$-deformation is radial. This is closely related to the fact that the $q$-deformed Dirac operator is invariant under the spin group as will be proven in the following section, and not under a $q$-deformed version of the spin group.

The action of the $q$-Dirac operator can be extended to general radial functions, not necessarily in $\mathcal{P}$. When $f$ is a scalar function, axiom (B3) can be extended to

$$
\partial_{\underline{x}}^{q} f\left(\underline{x}^{2}\right)=-\frac{x}{r}\left(D_{r}^{q} f\left(\underline{x}^{2}\right)\right)+f\left(q^{2} \underline{x}^{2}\right) \partial_{\underline{x}}^{q} .
$$

The space of all scalar radial functions for which the expression above exists is denoted by $\mathcal{J}$.

\subsection{Unicity of the $q$-Dirac operator on Clifford analysis}

Theorem 5. The Dirac operator $\partial_{\underline{x}}^{q}$ on $\mathcal{P}=\mathbb{R}\left[x_{1}, \cdots, m\right] \otimes \mathbb{C}_{m}$ in formula (20) is uniquely determined by the following list of properties:

$$
\begin{aligned}
& \partial_{\underline{x}}^{q}(\underline{x})=[m]_{q} \\
& \partial_{\underline{x}}^{q} \underline{x}^{2}=q^{2} \underline{x}^{2} \partial_{x}^{q}+(q+1) \underline{x} \\
& \left(\partial_{\underline{x}}^{q} x+q x \partial_{\underline{x}}^{q}\right) \text { is a scalar operator } \\
& \partial_{\underline{x}}^{q} \text { is Spin }(m) \text {-invariant } \\
& \mathbb{E} \partial_{\underline{x}}^{q}=\partial_{\underline{x}}^{q}(\mathbb{E}-1) .
\end{aligned}
$$


Proof. First, it can be checked that the $q$-Dirac operator in equation (20) satisfies these properties. Properties $(C 0)$ and $(C 7)$ are trivial. Properties $(C 3)$ and $(C 5)$ follow from equation $(21)$. Property $(C 6)$ holds since $\underline{x}, \partial_{\underline{x}}$ and $\mathbb{E}$ are $\operatorname{Spin}(m)$-invariant.

In the exact same way as in the proof of lemma 2 properties $(C 0),(C 3)$ and (C5) imply that equation (21) must hold. The action on a polynomial of the form $\underline{x}^{l} M_{k}$ with $M_{k} \in \mathcal{M}_{k}$ is therefore uniquely determined when $\partial_{\underline{x}}^{q} M_{k}$ is determined.

Axiom $(C 7)$ implies that the image of $\partial_{\underline{x}}^{q}$ acting on the space $\mathcal{M}_{k}$ is a subspace of $\mathcal{P}_{k-1}$. As a $\operatorname{Spin}(m)$-representation $\mathcal{M}_{k}$ decomposes into irreducible pieces as (for simplicity we consider the case $m=2 n+1$ )

$$
\mathcal{M}_{k}=\bigoplus_{i=0}^{2^{n}} \mathcal{M}_{k}^{i}
$$

Each $\mathcal{M}_{k}^{i}$ is an irreducible highest weight representation for $\operatorname{Spin}(m)$, with highest weight $\left(k+\frac{1}{2}, \frac{1}{2}, \cdots, \frac{1}{2}\right)$, where the term $\frac{1}{2}$ is repeated $n-1$ times. This essentially follows from the decomposition of $\mathbb{C}_{m}$ into its spinor spaces. The decomposition of the space $\mathcal{P}_{k-1}$ in lemma 1 ,

$$
\mathcal{P}_{k-1}=\bigoplus_{j=0}^{k-1} \underline{x}^{j} \mathcal{M}_{k-1-j}
$$

implies that there does not appear a $\operatorname{Spin}(m)$-representation of highest weight $\left(k+\frac{1}{2}, \frac{1}{2}, \cdots, \frac{1}{2}\right)$ in $\mathcal{P}_{k-1}$. Since the $q$-Dirac operator is $\operatorname{Spin}(m)$-invariant and the image of $\partial_{\underline{x}}^{q}$ acting on the space $\mathcal{M}_{k}$ is inside $\mathcal{P}_{k-1}$, we find that $\partial_{\underline{x}}^{q}$ must be zero on $\mathcal{M}_{k}^{\underline{x}}$. The case $m=2 n$ is completely equivalent.

Summarizing, the axioms imply that $\partial_{x}^{q} \underline{x}^{l} M_{k}$ is given by the expressions in theorem 4 which implies that $\partial_{\underline{x}}^{q}$ is uniquely determined and corresponds to the expression in equation (20).

\section{5. $q$-integration}

Since the deformation is purely radial, it is logical to define integration as a combination of $q$-deformed (Jackson) radial integration with undeformed spherical integration, $\int_{\mathbb{S} m-1} d \sigma$, as was also done in [4]. The spherical integration satisfies $\int_{\mathbb{S} m-1} d \sigma \underline{x} \mathcal{M}_{k}=0$ for $k \in \mathbb{N}$ and $\int_{\mathbb{S} m-1} d \sigma \mathcal{M}_{k}=0$ for $k>0$. We define integration for functions of the form $\mathcal{P} \otimes \mathcal{J}$ with $\mathcal{J}$ the scalar radial functions such that the $q$-Dirac operator is defined on them.

Definition 2. The q-integration on $\mathcal{P} \otimes \mathcal{J}$ is defined as

$$
\int_{\mathbb{B}^{m}(\lambda)} f(\underline{x}) d_{q} V(\underline{x})=\int_{0}^{\lambda} d_{q} r r^{m-1} \int_{\mathbb{S}^{m-1}} d \sigma f(\underline{x}),
$$

with the one dimensional q-integration as defined in equation (4) for $\lambda \in \mathbb{R}^{+}$ or equation (5) for $\lambda=a \cdot \infty$ with $a \in \mathbb{R}^{+}$. 
The vector $\underline{\xi}$ will be used for the normalized vector variable, $\underline{\xi}=\underline{x} / r$. The integration over the boundary of $\mathbb{B}^{m}(\lambda)$, the ball with radius $\bar{\lambda}$ in $\mathbb{R}^{m}$, is denoted by $\int_{\partial \mathbb{B}^{m}(\lambda)} d \sigma$ and satisfies

$$
\int_{\partial \mathbb{B}^{m}(\lambda)} d \sigma f(\underline{x})=\lambda^{m-1} \int_{\mathbb{S}^{m-1}} d \sigma f(\lambda \underline{\xi}) .
$$

Now we prove that Stokes' theorem holds for this $q$-integration and the $q$-Dirac operator.

Theorem 6. For $f \in \mathcal{P} \otimes \mathcal{J}$ and the integration in defintion 2 , the following relations hold,

$$
\begin{aligned}
\int_{\mathbb{B}^{m}(\lambda)}\left(\partial_{\underline{x}}^{q} f\right) d_{q} V(\underline{x})= & -\int_{\partial \mathbb{B}^{m}(\lambda)} d \sigma \underline{\xi} f(\underline{x}) \quad \text { if } \lambda \in \mathbb{R}^{+} \\
= & 0 \quad \text { if } \lambda=a \cdot \infty, a \in \mathbb{R}^{+} \\
& \quad \text { and } \lim _{k \rightarrow \infty} \int_{\partial \mathbb{B}^{m}\left(q^{-k} a\right)} d \sigma \underline{\xi} f=0 .
\end{aligned}
$$

Proof. The space $\mathcal{P} \otimes \mathcal{J}$ is generated by functions of the form $M_{k} g\left(\underline{x}^{2}\right)$ and $\underline{x} M_{k} g\left(\underline{x}^{2}\right)$ with $M_{k} \in \mathcal{M}_{k}$ and $g\left(\underline{x}^{2}\right)$ a scalar valued radial function. It is immediately clear that both the left-hand and right-hand side of the proposed equations are zero unless $k=0$. The first possibility we must check is therefore $f(\underline{x})=g\left(\underline{x}^{2}\right)$. Equation (22) shows that the left-hand side is equal to

$$
\int_{\mathbb{B}(\lambda)}\left(\partial_{\underline{x}}^{q} f\right) d_{q} V(\underline{x})=-\int_{\mathbb{B}(\lambda)} \frac{\underline{x}}{r}\left(D_{r}^{q} g\left(\underline{x}^{2}\right)\right) d V(\underline{x}) .
$$

Therefore both left-hand and right-hand side are zero. The only case left is $f(\underline{x})=\underline{x} g\left(\underline{x}^{2}\right)$. The left-hand side can be calculated using equations (21) and $(22)$ as

$$
\begin{aligned}
& \int_{\mathbb{B}(\lambda)}\left(\partial_{\underline{q}}^{q} f\right) d_{q} V(\underline{x}) \\
= & {[m]_{q} \int_{\mathbb{B}(\lambda)} g\left(\underline{x}^{2}\right) d_{q} V(\underline{x})+\left(q^{m}+q\right) \int_{\mathbb{B}(\lambda)} r D_{r}^{q} g\left(\underline{x}^{2}\right) d_{q} V(\underline{x}) } \\
+ & q \int_{\mathbb{B}(\lambda)} \frac{\underline{x}^{2}}{r} D_{r}^{q} g\left(\underline{x}^{2}\right) d_{q} V(\underline{x}) \\
= & {[m]_{q} \int_{\mathbb{B}(\lambda)} g\left(\underline{x}^{2}\right) d_{q} V(\underline{x})+q^{m} \int_{\mathbb{B}(\lambda)} r D_{r}^{q} g\left(\underline{x}^{2}\right) d_{q} V(\underline{x}) . }
\end{aligned}
$$

In case $\lambda \in \mathbb{R}^{+}$, the following relation can be calculated using equations (3) and (6)

$$
\begin{aligned}
q^{m} \int_{0}^{\lambda} d_{r} r^{m} D_{r}^{q} h(r) & =\int_{0}^{\lambda} d_{q} r D_{r}^{q}\left(r^{m} h(r)\right)-[m]_{q} \int_{0}^{\infty} d_{q} r r^{m-1} h(r) \\
& =\lambda^{m} h(\lambda)-[m]_{q} \int_{0}^{\infty} d_{q} r r^{m-1} h(r) .
\end{aligned}
$$


For $\lambda \in \mathbb{R}^{+}$we therefore obtain

$$
\begin{aligned}
\int_{\mathbb{B}(\lambda)}\left(\partial_{\underline{x}}^{q} f\right) d_{q} V(\underline{x}) & =\lambda^{m} g\left(-\lambda^{2}\right) \int_{\mathbb{S}^{m-1}} d \sigma 1 \\
& =-\int_{\partial \mathbb{B}^{m}(\lambda)} d \sigma \underline{\xi} \underline{x} g\left(\underline{x}^{2}\right)=-\int_{\partial \mathbb{B}^{m}(\lambda)} d \sigma \underline{\xi} f(\underline{x}) .
\end{aligned}
$$

The infinite integration can be calculated in the exact same way. It can also be obtained from the first property by observing the relation $\int_{0}^{a \cdot \infty} d_{q} r=$ $\lim _{k \rightarrow \infty} \int_{0}^{q^{-k} a} d_{q} r$.

\section{Orthogonal polynomials}

Now that the integration corresponding to the $q$-Dirac operator is defined, one can consider orthogonal polynomials. Therefore we consider a fixed basis $\left\{M_{k}^{(l)}\right\}$ for the space of spherical monogenics $\mathcal{M}_{k}$ which satisfies

$$
\int_{\mathbb{S}^{m-1}} d \sigma\left[\overline{M_{k}^{(l)}} M_{k}^{(t)}\right]_{0}=\delta_{l t},
$$

with $[\cdot]_{0}: \mathbb{C}_{m} \rightarrow \mathbb{C}$ the projection of the Clifford algebra onto its scalar part. In theorem 10 in [4] it was proven that for the inner product on $\mathcal{P}$ defined as

$$
\langle f \mid g\rangle=\int_{\mathbb{B}^{m}\left(\frac{1}{\sqrt{1-q^{2}}}\right)}[\overline{f(\underline{x})} g(\underline{x})]_{0} e_{q^{2}}\left(\underline{x}^{2}\right) d_{q} V(\underline{x}),
$$

the $q$-Clifford-Hermite polynomials given by

$$
\phi_{j, k, l}= \begin{cases}\mathcal{L}_{i}^{\frac{m}{2}+k-1}\left(-\underline{x}^{2} \mid q^{2}\right) M_{k}^{(l)} & \text { if } j=2 i \\ \underline{x} \mathcal{L}_{i}^{\frac{m}{2}+k}\left(-\underline{x}^{2} \mid q^{2}\right) M_{k}^{(l)} & \text { if } j=2 i+1,\end{cases}
$$

form an orthogonal basis for $\mathcal{P}$. Although these polynomials are orthogonal for the integration connected to the $q$-Dirac operator, they do not behave well with respect to the $q$-Dirac operator developed in the present paper. For instance it can be calculated that the relation

$$
\partial_{\underline{x}}^{q} \phi_{j, k, l}=C_{j, k, l} \phi_{j-1, k, l}
$$

will not hold for any constants $C_{j, k, l}$. Such an equation does hold for the $q$ Dirac operator developed in [4], which therefore is better suited to generalize the theory of $q$-orthogonal polynomials (see e.g. $[3,12]$ ) to Clifford analysis. Such an equation also holds for the $q$-Dirac operator developed in the present paper and the polynomials $\psi_{j, k, l}$ defined as

$$
\psi_{j, k, l}= \begin{cases}\mathcal{L}_{i}^{\alpha_{k, m}-1}\left(-\underline{x}^{2} \mid q^{2}\right) M_{k}^{(l)} & \text { if } j=2 i \\ \underline{x} \mathcal{L}_{i}^{\alpha_{k, m}}\left(-\underline{x}^{2} \mid q^{2}\right) M_{k}^{(l)} & \text { if } j=2 i+1\end{cases}
$$

with $\alpha_{k, m}$ satisfying $\left[2 \alpha_{k, m}\right]_{q}=[k+m]_{q}+q[k]_{q}$. However, these polynomials are not orthogonal with respect to the inner product introduced above. 


\section{Conclusion}

The $q$-Dirac operator defined by the list of axioms $(B 0)-(B 7)$ is more naturally defined compared to the Dirac operator in [4]. In the case of Clifford analysis the $q$-Dirac operator in the current paper is uniquely defined from the axioms $(C 0),(C 3),(C 5),(C 6)$ and $(C 7)$. The real difference between this list and the list of axioms $(A 1)-(A 4)$ on p7 of [4] is that in the present paper the $q$-anticommutator of $\partial_{x}^{q}$ and $\underline{x}$ has to be scalar while in [4] the square of the $q$-Dirac operator has to be scalar. The first condition is logical in the framework of Clifford analysis, while the second condition is necessary in order to obtain a $q$-deformed Laplace operator.

Both $q$-Dirac operators satisfy Stokes' formula for the same type of $q$ integration on $\mathbb{R}_{q}^{m}$. However, as the theory of $q$-Clifford-Hermite polynomials, which are orthogonal with respect to this integration, is more closely linked to the $q$-Dirac operator in [4], that operator is better suited to study $q$ polynomials.

There are still other possible lists of axioms which define $q$-Dirac operators. Each definition seems to have its own specific advantage with respect to integration, the Fischer decomposition, axial functions or $q$-polynomials. An important direction for further research is a systematic study of the link between changes in the axioms and properties of the resulting $q$-Dirac operator.

\section{Acknowledgment}

K. Coulembier is supported by a Ph.D. Fellowship of the the Research Foundation - Flanders (FWO).

\section{References}

[1] F. Brackx, R. Delanghe and F. Sommen, Clifford analysis, vol. 76 of Research Notes in Mathematics. Pitman (Advanced Publishing Program), Boston, MA, 1982.

[2] U. Carow-Watamura, M. Schlieker, S. Watamura and W. Weich, Bicovariant differential calculus on quantum groups $\mathrm{SU}_{q}(N)$ and $\mathrm{SO}_{q}(N)$. Comm. Math. Phys. 142 (1991) no. 3, 605-641.

[3] N. Ciccoli, E. Koelink and T. Koornwinder, $q$-Laguerre polynomials and big $q$-Bessel functions and their orthogonality relations. Methods Appl. Anal. 6 (1999) no. 1, 109-127.

[4] K. Coulembier and F. Sommen, $q$-deformed harmonic and Clifford analysis and the $q$-Hermite and Laguerre polynomials. J. Phys. A: Math. Theor. 43 (2010) 115202.

[5] H. De Bie, B. Orsted, P. Somberg and V. Soucek, Dunkl operators and a family of realizations of $\operatorname{osp}(1 \mid 2)$. arXiv:0911.4725.

[6] H. De Bie and F. Sommen, Correct rules for Clifford calculus on superspace. Adv. Appl. Clifford Algebr. 17 (2007) no. 3, 357-382. 
[7] H. De Bie and F. Sommen, Fischer decompositions in superspace. Function spaces in complex and Clifford analysis. National University Publishers, Hanoi (2008) pp. 170-188.

[8] R. Delanghe, F. Sommen and V. Souček, Clifford algebra and spinor-valued functions, vol. $\mathbf{5 3}$ of Mathematics and its Applications. Kluwer Academic Publishers Group, Dordrecht, 1992.

[9] H. Exton, q-hypergeometric functions and applications. Ellis Horwood, Chichester, 1983.

[10] G. Gasper and M. Rahman, Basic hypergeometric series. Encyclopedia of Mathematics and its Applications, 35. Cambridge University Press, Cambridge, 1990.

[11] A. KempF and S. MAJID, Algebraic $q$-integration and Fourier theory on quantum and braided spaces. J. Math. Phys. 35 (1994) no. 12, 6802-6837.

[12] R. Koekoek, P.A. Lesky and R.F. SwartTouw, Hypergeometric orthogonal polynomials and their q-analogues. Springer Monographs in Mathematics. Springer-Verlag, Berlin, 2010.

[13] F. Jackson, On $q$-functions and a certain diffrence operator. Trans. Roy. Soc. Edin. 46 (1908) 253-281.

[14] D. Monk, The q-analogue of the Laguerre polynomials. J. Math. Anal. Appl. 81 (1981) no. 1, 20-47.

[15] F. Sommen, An algebra of abstract vector variables. Portugaliae Math. 31, Vol. 54, Fasc. 3 (1997) 287-310.

K. Coulembier

Krijgslaan 281

9000 Gent

Belgium

e-mail: coulembier@cage.ugent.be

F. Sommen

Krijgslaan 281

9000 Gent

Belgium

e-mail: fs@cage.ugent.be 